Annuaire suisse de politique de développement

4 | 1984

Annuaire Suisse - Tiers Monde 1984

\title{
Bibliographie
}

Literaturverzeichnis

\section{Antoine Brawand}

\section{CpenEdition}

\section{Journals}

Édition électronique

URL : http://journals.openedition.org/aspd/1178

DOI : $10.4000 /$ aspd. 1178

ISSN : 1663-9669

\section{Éditeur}

Institut de hautes études internationales et du développement

\section{Édition imprimée}

Date de publication : 1 janvier 1984

Pagination : 212-230

ISSN : 1660-5934

\section{Référence électronique}

Antoine Brawand, «Bibliographie », Annuaire suisse de politique de développement [En ligne], 4 | 1984 mis en ligne le 03 février 2013, consulté le 08 septembre 2020. URL : http://journals.openedition.org/ aspd/1178; DOI : https://doi.org/10.4000/aspd.1178 


\section{Bibliographie Literaturverzeichnis}

Antoine Brawand 


\section{INTRODUCTION}

La bibliographie contient les références de textes qui ont paru depuis septembre 1983. Le dépouillement a été arrêté à fin août 1984.

Les références indiquées dans la bibliographie (1) concernent spécifiquement les relations Suisse - Tiers Monde. Lors de la sélection des textes, nous avons également trouvé des écrits d'intérêt général ou consacrés à d'autres thèmes, qui abordent aussi le sujet, de façon explicite ou implicite. Dans ces cas, vu I'abondance de la matière et l'existence d'autres bibliographies (2), nous avons seulement retenu les références des textes qui traitent explicitement, dans des passages importants, des rapports entre la Suisse et le Tiers Monde.

Dans la collecte de l'information, j'ai bénéficié de précieux concours.

Mes remerciements s'adressent particulièrement au Service d'information Tiers Monde $(\mathrm{i} 3 \mathrm{~m})$ à Berne. J'exprime également ma reconnaissance à la Direction de la coopération au développement et de l'aide humanitaire, à I'Office fédéral des affaires économiques extérieures, à la Société suisse de statistique et d'économie politique, à la bibliothèque de I'IUED et à tous ceux qui ont bien voulu répondre à mes demandes de renseignements. $\mathrm{J}$ 'assume toutefois la responsabilité de la sélection et de la classification des références qui figurent dans la bibliographie.

\section{A.B.}

Note : Les documents publiés en allemand et en français par un même auteur, un même éditeur et à la même date sont indiqués selon leur titre français, suivi du sigle (All.). Les publications ne remplissant pas ces trois conditions sont mentionnées dans chaque langue. Le titre est également suivi d'un sigle (All. ou Fr. selon le cas).

1. A l'exclusion des articles de journaux. Quelques titres omis dans les premiers Annuaires sont ajoutés ici.

2. Voir notamment la "Bibliographie suisse de science politique", qui paraît dans l'Annuaire suisse de science politique, et la Bibliographie suisse de statistique et d'économie politique, qui paraît tous les deux ans. 


\section{CLASSIFICATION}

Numéros de référence

I. Personnes
A. Ouvrages
B. Articles, discours, etc.

1- 43

44-132

$133-223$
A. Confédération suisse
133-170b
B. Institutions privées
$171-223$

Note :

La distinction entre les deux grands groupes d'auteurs a pour but de simplifier la présentation. Les publications de la Confédération ou des organisations privées, signées par des personnes (il s'agit surtout d'articles et de discours), figurent sous la première rubrique.

\section{ABREVIATIONS ET SIGNES}

(All.) Document disponible également en allemand.

(Fr.) Document disponible également en français.

FF Feuille fédérale

Indication suivie de l'année, du numéro du volume et du numéro de la première page. La version allemande des textes figure dans le Bundesblatt (même année et même numéro de volume).

RO Recueil des lois fédérales Indication suivie de l'année et du numéro de la première page. La version allemande des textes se trouve dans la Samm/ung der eidgenössischen Gesetze.

EDA Eidgenössisches Departement für auswärtige Angelegenheiten.

DEH Direktion für Entwicklungszusammenarbeit und humanitäre Hilfe.

EVD Eidgenössisches Volkswirtschaftsdepartement.

BAWI Bundesamt für Aussenwirtschaft.

DFAE Département fédéral des affaires étrangères.

DDA Direction de la coopération au développement et de l'aide humanitaire.

DFEP Département fédéral de l'économie publique.

OFAEE Office fédéral des affaires économiques extérieures. 


\section{PERSONNES}

\section{A. Ouvrages}

1. BALMER D. - L'Heure du cuivre. Lausanne, Ed. d'en bas, 1984, $160 \mathrm{p}$.

2. BORNER S., BURGENER, B., STUCKEY B. und WEHRLE F. - Die Internationalisierung der Industrie und die Schweiz als Unternehmungsstandort. Basel, Institut für Sozialwissenschaften / Institut für angewandte Wirtschaftsforschung, Diskussionspapiere, 1983.

3. BORNER S. und WEHRLE F. - Die Sechste Schweiz : Ueberleben auf dem Weltmarkt. Zürich, Orell Füssli Verlag, 1984.

4. BRAUEN M. - Nepal-Leben und Ueberleben. Zürich, Völkerkundemuseum der Universität, 1983, $245 \mathrm{~S}$.

5. BRUGGMANN H. - Ein Plan zur Erhöhung des Ressourcentransfers in die Entwicklungs/änder zum Nutzen von Entwicklungs-, OEPC- und Industrieländern. Diessenhofen, Verlag Rüegger (Reihe Weltwirtschaft, Bd 5), 1983, 352 S.

6. BRUNNER u.a. - Einblick in die schweizerische Aussenpolitik. Zum 65. Geburtstag von Staatssekretär Raymond Probst. Zürich, Verlag Neue Zürcher Zeitung, 1984, $469 \mathrm{~S}$.

7. BUECHLER P. - La misère des bidonvilles : un défi à relever. Aide aux bidonvilles : fondements et propositions. Lucerne, Ed. Caritas, 1983, 32 p.

8. CAMPBELL B.K. - Les enjeux de la bauxite. La Guinée face aux multinationales de I'aluminium. Genève, Presses de I'Université de Montréal / Institut universitaire de hautes études internationales, Diffusion I'Harmattan, 1983, 182 p.

9. DUVAL J.-F., GRENON J.-P. et LEGERET J. - Des Suisses pas comme les autres et au bout de la terre. Zurich/Genève, Construire et Ed. de I'Aire, 1983, $216 \mathrm{p}$.

10. EIGEL W. - Entwicklung und Menschenrechte. Entwicklungszusammenarbeit am Horizont der Menschenrechte. Freiburg, IMBA Verlag, 1983, 340 S.

11. FOURNIER I. - L'aide aux ressortissants du Tiers-Monde en Suisse romande : la situation des étudiants et des "passants". Lucerne, Caritas Suisse (Documentation 1984/1), mai 1984, 24 p.

12. FREI D. (Hrsg.) - Wege aus der Weltwirtschaftskrise. Diessenhofen, Verlag Rüegger (Sozialwissenschaftliche Studien für das Schweizerische Institut für Auslandforschung Zürich, Band 13), 1984, $140 \mathrm{~S}$.

13. FRIEDEN J. - Le Tiers Monde entre la dépendance et l'indépendance monétaire : réflexion sur les paiements internationaux, le transfert de ressources et les conditions extérieures du développement. Fribourg, Ed. Universitaires (Documents économiques), 1983, $294 \mathrm{p}$.

14. HAEGLER R.P. - Schweizer Universalismus, UNO Partikularismus. Bern, Peter Lang Verlag, 1983, $273 \mathrm{~S}$.

15. HANSELMANN G. - La fuite des capitaux du tiers monde. Réalité et fiction. Zurich, Union de Banques Suisses, 1984, 15 p. (All., Italien.) 
16. HAUSER J.A. und RAMBOUSEK W.H. - Private Enterprise and Development : The Case of the Santa Monica Plant of Hindustan Ciba-Geigy Ltd, IBH Publishing Co, Bombay and London, 1984.

17. GUT R.E. - Initiative contre les banques - vers une votation cruciale. Genève, Société pour le développement de l'économie suisse (SDES), 1984, 24 p. (All.)

18. IMFELD A. - Zucker. Zürich, Unionsverlag, 1983, $200 \mathrm{~S}$.

19. JACOB J.-P. et BAUMANN H. - Stratégie des développeurs et tactiques des développés : projet de Caritas Suisse dans le Callejon de Huaylas, Pérou. Genève, IUED (Coll. Itinéraires, No 29), 1983, 158 p.

20. JAEGGI M. - Mehr als ein Konto. Bankwesen und Bankengesetz : Reformen in Richtung Ausgleich und Entwicklung ? Basel, Brot für Brüder, 1984, 55 S. (Fr.)

21. JAGGI Y. - Politique économique extérieure. Défense nationale économique. Lausanne, Presses polytechniques romandes (Coll. Droit et vie économique), 1983, $93 \mathrm{p}$.

22. JOLLES P.R. - Von der Handelspolitik zur Aussenwirtschaftspolitik. Ausgewählte Reden und Aufsätze. Bern, Stämpfli, 1983, 403 S.

23. JON I. - La coopération au développement : un débat en Suisse dans la première moitié des années 1970 (Elaboration d'une loi fédérale sur la coopération au développement et l'aide humanitaire internationales 1971-1976). Mémoire de licence, Section d'histoire contemporaine, Faculté des Lettres, Université de Genève, mars 1984, 73 p. plus annexes.

24. KAUFMANN O.K., KOLLER A. und RIKLIN A. (Hrsg.) - Zur Zukunft von Staat und Wirtschaft in der Schweiz. Festschrift für Bundesrat Dr. Kurt Furgler zum 60. Geburtstag. Zürich, Benziger, 1984, 358 S.

25. KERN E.L. - Situative Ausbildung für Entwicklungs/änder : theoretische Grundlagen und saudiarabische Erfahrungen. Diss. Wirtschaftswiss., St. Gallen, 1983, XIV, 482 S.

26. KREUER W. - Entwicklungs/änder, Entwicklungshilfe, Entwicklungspolitik. Frankfurt a.M., M. Diesterweg O. Salle, Aarau, Sauerländer, 1984, 191 S.

27. KRIPPENDORF J. - Die Ferienmenschen. Für ein neues Verständnis von Freizeit und Reisen. Zürich, Verlag Orell Füssli, 1983, 244 S.

28. LEMOINE M. - Bitterer Zucker. Zürich, Rotpunkt-Verlag, 1983, 273 S.

29. LETSCH R. - Rhodesien, die Vereinten Nationen und die Schweiz. Konzepte und Inkonsistensen der Rhodesienpolitik. Diss. Wirtschafts- und Sozialwissenschaften, St. Gallen, 1983, 427 S.

30. MIROW K.R. - La dictature des cartels. (Trad. de I'espagnol.) Grenoble, Presses universitaires de Grenoble, 1982, 207 p.

31. MUELLER E. - Das Gatt und die Entwicklungs/änder. Bern, Peter Lang Verlag, $1983,118 \mathrm{~S}$.

32. OBERHAENSLI H. - Internationale Rohstoffpolitik im Zeichen von Instabilität und Inflation. Diessenhofen, Verlag Rüegger, 1982, $581 \mathrm{~S}$. 
33. PERROT A. - Un défi à relever par les défenseurs des droits de l'homme et du tiers-monde. Réponse aux propos démobilisants. Tirage à part, revu et complété, d'une série d'articles parus dans Le Courrier au premier semestre de 1983. Genève, Le Courrier, 1984, $20 \mathrm{p}$.

34. PREISWERK R. - A contre-courants. L'enjeu des relations interculturelles. Textes réunis et publiés par G. Rist. Lausanne, Ed. d'en bas (Coll. Nord-Sud), 1984,258 p.

35. ROSSEL P. - Tourisme et Tiers Monde : un mariage blanc. Lausanne, Ed. P.-M. Favre (Coll. Centre Europe - Tiers Monde), 1984.

36. SCHMASSMANN N. - Verteilungswirkungen von Erdölpreissteigerungen. Versuch einer empirischen Analyse für die Schweiz 1972-1980. (Diss.) Diessenhofen, Rüegger (Basler Sozialökonomische Studien, Nr. 21), 1983, 326 S.

37. STRAUBHAAR T. - Arbeitskräftewanderung und Zahlungsbilanz. Eine empirische Untersuchung am Beispiel der Rücküberweisungen nach Griechenland, Portugal, Spanien und der Türkei. Bern, Verlag P. Haupt (Berner Beiträge zur Nationalökonomie, Bd 45), 1983, 229 S.

38. SULTAN M. et TAVIER C. - La Suisse sous verre. Ou I'image de la Suisse et du Suisse que se font les fonctionnaires internationaux du Tiers Monde. Genève, Département de sociologie de I'Université, Mémoire de licence, déc. 1983, ronéoté.

39. WAGNER A. und BEUTTER F. - Finanzplatz Schweiz - Dritte Welt. Freiburg, IMBA Verlag, 1983, $140 \mathrm{~S}$.

40. WEHRLE F. - Veränderung der weltwirtschaftlichen Rahmenbedingungen und die Internationalisierung der Schweizer Industrie. Basel, Institut für Sozialwissenschaften / Institut für angewandte Wirtschaftsforschung, Diskussionspapier Nr. 2, 1983, ca. $136 \mathrm{~S}$.

41. WEHRLE F. - Der exportorientierte Industrialisierungsprozess in den Schwellenländern Asiens und Lateinamerikas und Japans. Aufstieg zur Wirtschaftsgrossmacht Betrachtungen aus einer schweizerischen Perspektive. Basel, Institut für Sozialwissenschaften / Institut für angewandte Wirtschaftsforschung, Diskussionspapier Nr. 3, 1983, ca. $118 \mathrm{~S}$.

\section{Autres ouvrages}

42. Internationale Wirtschafts- und Finanzpolitik in der Herausforderung der achtziger Jahre. Aussenwirtschafts-Beiheft Nr. 1, 1984.

43. Schwellenländer : Marktpartner und Konkurrenten der schweizerischen Industrie. Informations-Tagung vom 3.11.1983 in Zürich (HSG-Weiterbildungsstufe). St. Gallen, Hochschule für Wirtschafts- und Sozialwissenschaften, 1983. Dossier ca. $100 \mathrm{~S}$. 
B. Articles, discours, etc.

44. ALBONICO H. - "Ciba-Geigy in Simbabwe. Fragwürdige Agroberatung." Mosquito, Nr. 2, März 1984, S. 20.

45. ARIOLI S. - "Exportfinanzierung und Internationale Verschuldung." Vortrag vor der Volkswirtschaftlichen Gesellschaft des Kantons Bern, 14.11.83. Bern, BAWI, 26 S.

46. BACHEM M. u.a. - "Monetäre Bedrohungsformen im Rahmen der Sicherheitspolitik : Die internationale Verschuldung aus der Sicht der Schweiz." Studien zur Sicherheitspolitik (Bern, Zentralstelle für Gesamtverteidigung), Nr. 1, 1984, $32 \mathrm{~S}$.

47. BAILLOD G. - "Point de vue." Dans : Horlogerie suisse et Tiers Monde. Lausanne, Service d'information Tiers Monde (i3m), Dossier No 14, mai 1984, pp. 69-71.

48. BAUER G. - "Point de vue." Dans : Horlogerie suisse et Tiers Monde. Lausanne, Service d'information Tiers Monde (i3m), Dossier No 14, mai 1984, pp. 61-68.

49. BAUER T. - “Verschuldung und Kapitalflucht. Teufelsspirale für die Dritte Welt." Solidarität, Nr. 83, Dezember 1983, S. 10-12.

50. BAUMANN M. - "Diktator Mobutu bei Bundesrat Aubert." Südwind, Nr. 4, Juli 1983, S. 17-18.

51. BAUMANN M. - "Saatgut-Multis. 45 \% Gewinn." Mosquito, Nr. 1, Januar 1984, S. 22-23.

52. BAUMANN M. - "Sri Lanka. Nestlé in der ersten Agrarfreihandelszone." Südwind, Nr. 4, Juli 1983, S. 9.

53. BAUMGARTNER H.J. - “Hilfswerk 'mediswiss' : Ciba-Geigy's barmherzige Schwester." Mosquito, Nr. 1, Januar 1984, S. 10-12.

54. BAUMGARTNER H.J. - "Malaria-Resistenz rentiert." Südwind, Nr. 8, Dezember 1983, S. 11-13.

55. BAUMGARTNER H.J. - "Pharmaforschung für die Dritte Welt : Ausser Spesen..." Südwind, Nr. 5/6, September 1983, S. 14-19.

56. BLANC J.-F. - "Crise et restructuration horlogère : Quels enjeux ? Pour quel développement ?" Dans : Horlogerie suisse et Tiers Monde, Lausanne, Service d'information Tiers Monde (i3m), Dossier No 14, mai 1984, pp. 5-60.

57. BOESCH I. et al. - "IKRK - Visitenkarte der Schweiz. In jedem Fall neutral." Mosquito, Nr. 3/4, Mai 1984, S. 4-9.

58. BOLLMANN F. - "Les projets de coopération technique sont-ils productifs ?" Entwicklung/Développement, No 17, juillet 1984, pp. 40-43. (All.)

59. BOENI R.T. - "International Indebtedness and the Swiss Banks." Economical and Financiel Prospects (Basle, Swiss Bank Corporation), No 3, June/July 1984, pp. 5-7.

60. BRAWAND A. - "Statistiques du commerce, des flux financiers et de l'aide publique au développement." Annuaire Suisse - Tiers Monde / Jahrbuch Schweiz Dritte Welt 1983. Genève, IUED, No 3, 1983, pp. 283-359. 
61. BRENNER G. - "Seid umschlungen, Millionen. Wie Fluchtgeld in saubere Schweizer Fränkli verwandelt wird." Die Zeit-Magazin, Nr. 18, 27. April 1984, $7 \mathrm{~S}$.

62. BUSER E. - "Une réalisation commune de la chimie bâloise." Dans : "Coopération économique avec le Tiers Monde (VI", Bulletin, Crédit Suisse, février 1984, pp. 38-39.

63. CALOZ-TSCHOPP M.-C. - A propos des "nouveaux réfugiés" le pari de l'ouverture, Ed. Ligue suisse des droits de I'homme, Genève, 1984, $36 \mathrm{p}$.

64. CAMPICHE R.J. et PETER H.-B. - "Pratique de I'éthique sociale, ou peut-on moraliser le système bancaire suisse ?" Dans : Le Supplément, Revue d'éthique et de théologie morale, No 147, déc. 1983, pp. 449-568. Tiré à part : Berne/Lausanne, Institut d'éthique sociale de la FEPS, Texte IES, No 7, 1983, 13 p.

65. FINGER H. - "Schleichender Kurswechsel bei der Entwicklungshilfe ?" Reformatio, Heft 7/8, 1982, S. 391-402.

66. FORSTER J. - "Swiss Aid : Policy and Performance in European Developement Assistance." Vol. I. Policies and Performance, edited by Olaf Stokke, Norwegian Institute of International Affairs, Tilsburg and Oslo, EADI (Book Series 4), pp. 395-414.

67. FURGLER K. - "Wege aus der Krise : Die Stimme der Schweiz im Ringen um weltwirtschaftliche Lösungen." Ansprache am Hochschultag der Hochschule St. Gallen für Wirtschafts- und Sozialwissenschaften, 16.6.1984. Bern, EVD, $27 \mathrm{~S}$.

68. GEISSBUEHLER S., VOGT A. - "Bankeninitiative-Countdown läuft." Südwind, Nr. 7, Oktober 1983, S. 5-10.

69. GERBER J.-D. - “Wirtschafts- und Handelspolitische Massnahmen der Schweiz im Bereich der Entwicklungszusammenarbeit." Referat gehalten im Rahmen der Vortragsreihe der Reformierten Kirchgemeinde Küsnacht über "EntwicklungsHilfe, eine fragwürdige oder eine sinnvolle Sache ?" am 10.5.84. Bern, BAWI, $28 \mathrm{~S}$.

70. GERSTER R. - “20. Mai 1984 : Fluchtgeld Nein, Bankeninitiative Ja !" ArbeiterSolidarität, Nr. 21, März 1984, S. 12.

71. GONTARD J.-P., MABUT J.-F. et RAMELET C. - “'L'initiative ‘ $0,7 \%$ pour la création d'un fonds cantonal d'aide au développement'." Annuaire Suisse - Tiers. Monde / Jahrbuch Schweiz - Dritte Welt 1983. Genève, IUED, No 3, 1983, pp. 195-213.

72. GURTNER B. - "Schweizer Waffen für Pinochet." Südwind, Nr. 5/6, September 1983, S. 4-6.

73. GURTNER B. - "Ciba-Geigy : Neue Vorwürfe aus Brasilien." Südwind, Nr. 5/6, September 1983, S. 12.

74. GURTNER B. - "Schweiz : Durch die Hintertür in den IWF ?" Südwind, Nr. 5/6, September 1983, S. 32-33.

75. GURTNER B., MADOERIN K., MUGGLIN M. - "Dossier Brasilien - Schweiz." Mosquito, Nr. 3/4, Mai 1984, S. 17-22. 
76. HEIMO M.C. - Endettement extérieur du Tiers Monde et situation économique mondiale. Revue économique et sociale, 1984, No 2, pp. 129-144.

77. HOESLI E. - "L'aide au développement des communes et des cantons suisses." Annuaire Suisse - Tiers Monde / Jahrbuch Schweiz - Dritte Welt 1983. Genève, IUED, No 3, 1983, pp. 154-194.

78. HUBER T. - "Philippinen. Roche provoziert Boykott-Bewegung." Südwind, Nr. 5/6, September 1983, S. 34-37.

79. HUGI H.R. - “Angepasste Technologien für Entwicklungsländer." Schweizer Ing. u. Arch, 101, 1983, Nr. 50, S. 1193-1198.

80. HUERNI B. - “Der Beitritt der Schweiz zu den Institutionen von Bretton Woods : Wirtschaftliche Aspekte." Schweizerische Zeitschrift für Wissenschaft und Statistik, Juni 1984, S. 123-139.

81. JACOBI K. - “Die überseeischen Industrieländer als Absatzmärkte der Schweizer Exportindustrie." Referat gehalten vor der zehnjährigen Jubiläums-Generalversammlung der Kooperationsgemeinschaft Swissexport. Bern, BAWI, 30.9.1983, $22 \mathrm{~S}$.

82. JAEGGI M. - "Inter Press Service (IPS) : Le tiers monde sans voix ?" Entwicklung / Développement, No 16, décembre 1983, pp. 40-41. (All.)

83. JAEGGI M. et MUGGLIN M. - "Le paternalisme au lieu du dialogue : la Suisse et le débat sur le nouvel ordre international de I'information à I'UNESCO." Dans : Suisse, médias et Tiers-Monde. La Suisse face au nouvel ordre mondial de l'information et de la communication. Lausanne, Service d'information Tiers Monde (i3m), Dossier No 13, septembre 1983, pp. 1-23.

84. JENNY R. - "Swisscontact Entwicklungszusammenarbeit 1959-1984." Swisscontact 25, 1983, Nr. i4, S. 9-12.

85. JOLLES P. - "Die schweizerische Aussenwirtschaftspolitik : Erfahren und Ausblick." Zur Zukunft von Staat und Wirtschaft in der Schweiz. Festschrift für Bundesrat Dr. Kurt Furgler zum 60. Geburtstag. Hrsg. von O.K. Kaufmann, A. Koller und A. Riklin. Zürich, Benziger, 1984, S. 247-255.

86. JOLLES P. - "Aussenwirtschaft im Zeichen globaler Interdependenz." In : Internationale Wirtschafts- und Finanzpolitik in der Herausforderung der achtziger Jahre. Aussenwirtschafts- Beiheft Nr. 1, 1984.

87. KAMBER W. - “Caisses d'épargne et cautionnements - instruments de l'aide au développement." Dans : "Coopération économique avec le Tiers Monde (V)". Bulletin, Crédit Suisse, septembre 1983, pp. 40-41.

88. KAPPELER B. - "Point de vue." Dans : Horlogerie suisse et Tiers Monde. Lausanne, Service d'information Tiers Monde (i3m), Dossier No 14, mai 1984, pp. 73-75.

89. KELLER H. - "Chou En-Lai und unsere Beziehungen zur Volksrepublik China." Schweizerische Zeitschrift für Geschichte, Nr. 4, 1982, S. 574-580.

90. KOCH X. - "Asylrecht und Brot für die Flüchtlinge." Arbeiter-Solidarität, Nr. 22, Juni 1984, S. 6-8.

91. KUMMER R.D. - "China als Markt für die Schweizer Wirtschaft." Bern, BAWI, $1984,15 \mathrm{~S}$. 
92. LAAGER H. - “Flüchtlingshilfe als Beruf - ein Tagebuch mit Gedankenstrichen." Mitteilungsblatt Christlicher Friedensdienst, Nr. 365, September 1983, S. 9-14. (aus : Sozialarbeiter, Juli/August 1983.)

93. LEVY Ph. - “Die Handelspolitik der Schweiz gegenüber den Schwellenländern.” Referat anlässlich der Informationstagung (HSG-Weiterbildungsstufe) Schwe/lenländer - Marktpartner und Konkurrenten der schweizerischen Industrie der Hochschule St. Gallen für Wirtschafts- und Sozialwissenschaften am 3.11.83 in Zürich. Bern, BAWI, $20 \mathrm{~S}$.

94. LEVY Ph. - "Le transfert de technologie Nord-Sud vu par les pays exportateurs de technologie. La Suisse - un petit pays aux grands intérêts." Entwick/ung / Développement, No 16, 1983, pp. 24-26. (All.)

95. LEVY Ph. - "La promotion des investissements à I'étranger est-elle encore d'actualité ?" Exposé présenté à l'Assemblée générale de I'Union des Chambres de commerce suisses à l'étranger, le 17.5.84 à Lausanne. Berne, OFAEE, $9 \mathrm{p}$.

96. LEVY Ph. - “Déclaration générale de la délégation suisse à la $4 \mathrm{e}$ Conférence générale de l'ONUDI à Vienne", 2.8.1984, prononcée par Ph. Lévy. Berne, OFAEE, $8 \mathrm{p}$.

97. MADOERIN K. - "Ciba-Geigy. Expansion im Agrarsektor." Mosquito, Nr. 3/4, Mai 1984, S. 23-24.

98. MARTIN J. - "Les finances fédérales et I'aide publique au développement de la Suisse." Annuaire Suisse - Tiers Monde / Jahrbuch Schweiz - Dritte Welt 1983. Genève, IUED, No 3, 1983, pp. 215-226.

99. MAUCHER H. - "Probleme und Möglichkeiten in Schwellenländern - eine Beurteilung aus der Sicht eines multinationalen Unternehmens." In : Frei D. (Hrsg.), Wege aus der Weltwirtschaftskrise, Diessenhofen, Verlag Rüegger, 1984.

100. MORAND C. - "Les relations de la Suisse avec les institutions de Bretton Woods." Dans : Le Fonds Monétaire International et le Tiers Monde. Les 40 ans de Bretton Woods. Lausanne/Berne, Service d'information tiers monde (i3m), Dossier No 15, iuillet 1984, pp. 61-70.

101. MUGGLIN M. - "Mit Waffengeschäften gegen die Aermsten - Schweizer Waffen in Entwicklungsländern." In : Waffenplatz Schweiz. Beiträge zur schweizerischen Rüstungsindustrie und Waffenausfuhr. Hrsg. vom Tagungssekretariat "Für das Leben produzieren". Bern, Arbeitsgemeinschaft für Rüstungskontrolle und ein Waffenausfuhrverbot, 1983, S. 167-174.

102. MUGGLIN M. - "Verschuldung der Dritten Welt und die Schweiz. Geschäftsrezepte versagen." Solidarität, Nr. 79/80, Aug./Sept. 1983, S. 28-29.

103. MUGGLIN M. - "Finanzwelt und Schuldenkrise. Kurzsichtige Bankiers." Solidarität, Nr. 83, Dezember 1983, S. 7-9.

104. MUGGLIN M., SCHELBERT J. - “Neues Parlament. Im alten Trott.” Mosquito, Nr. 1, Januar 1984, S. 26-28.

105. PETER A. - "Theologisch-politische Rahmenüberlegungen zur Bankeninitiative." Neue Wege, Nr. 6, 1983, S. 165-168. 
106. PETER H.-B. - "Verschuldung der Entwicklungsländer - Banken - Sozialethik." Bern, Institut für Sozialethik des SEK, ISE-Texte Nr. 2, 1983, $10 \mathrm{~S}$. und Angestellten Revue des employés VSAM/FESM, Nr. 12, 1983, S. 368-371.

107. PETER T. - "Bankeninitiative und Entwicklungspolitik." Neue Wege, Nr. 3 , 1984, S. 90-93.

108. PREISWERK R. - “Les objectifs et les moyens d'une politique internationale suisse pour la paix." Dans : PREISWERK R., A contre-courants. L'enjeu des relations interculturelles. Textes réunis et publiés par G. Rist. Lausanne. Ed. d'en bas (Coll. Nord-Sud), 1984, pp.61-146.

109. PULT G. - "L'impact du Tiers Monde sur les postes de travail en Suisse." Annuaire Suisse - Tiers Monde /Jahrbuch Schweiz - Dritte Welt 1983. Genève, IUED, No 3 , 1983, pp. 227-245.

110. RENSCHLER R. - Der junge Alexander eroberte Indien. Er allein ? Die Vergewaltigung der Geschichte an unseren Schulen. Beiträge zur Entwicklungspolitischen Diskussion (Zürich, Erklärung von Bern), Nr. 14, Juli 1984, 33 S.

111. ROETLISBERGER E. - "Keine Einbahnstrasse." (Beitrag im Rahmen einer SHZ. Beilage über die Wirtschaftsbeziehungen Schweiz-Indien. Schweizerische Handelszeitung, Nr. 36, 1983, S. 41 und 43.

112. SCHAERER K. - "Verstetigung nach dem Boom ? Aktuelle Betrachtungen zur Entwicklung des Handels Schweiz-Iran." Vortrag an der Generalversammlung der Iranisch-schweizerischen Handels- und Industriekammer. Zürich, 6.7.1984. Bern, BAWI, $8 \mathrm{~S}$.

113. SCHELBERT J. - "Seerecht : Die Schweiz im Schlepptau der USA." Mosquito, Nr. 2, März 1984, S. 22-23.

114. SCHERRER C. - "Tourism is zero for Kenya." (deutsch) Südwind, Nr. 7, Oktober 1983, S. 15-17.

115. SCHMID H. - Jtaipu, der 'Singende Stein'. Der schweizerische Beitrag zur Fehlentwicklung Brasiliens. Beiträge zur Entwicklungspolitischen Diskussion (Zürich, Erklärung von Bern), Nr. 15, 1984.

116. SCHOENI W. - “Der schweizerische Kulturimperialismus und die UNESCO.” Widerspruch, Heft 6, November 1983, S. 95-104.

117. SIEBER H. - “Internationale Arbeitsteilung im Wandel. 'Alte Industriestaaten' - nicht zuletzt die Schweiz - unter Anpassungszwang." Der Staatsbürger, Nr. 4. 1984, S. 9-10.

118. SOMMARUGA C. - "Die Schweiz in der Weltwirtschaft von heute : Herausforderungen und aussenwirtschaftliche Prioritäten." Referat vor dem Schweizerischen Institut für Auslandforschung. Zürich, 26.6.84, Bern, BAWI, 26 S.

119. SOMMARUGA C. - "Pas d"'egoismo nationale della Svizzera'." Interview réalisée par P. Saladin. Entwicklung / Développement, No 17, juillet 1984, pp. 46-48. (All.)

120. STAEHELIN F.R. - "La coopération suisse au développement dans la crise économique internationale." Exposé présenté lors de la séance d'ouverture du cours postgrade sur les pays en développement de l'Ecole polytechnique fédérale de Lausanne, le 25.4.83. Bern, DFAE, 27 p. 
121. STAEHELIN F.R. - "Herausforderungen an Unternehmer und Entwicklungshelfer in der Dritten Welt." Swisscontact 25, 1983, Nr. 14, S. 4.

122. STETTER H., MARTIN J. et BESSON Ph. - "Revue des événements concernant les négociations internationales, la politique économique extérieure et la coopération au développement." Annuaire Suisse - Tiers Monde / Jahrbuch Schweiz Dritte Welt 1983. Genève, IUED, No 3, 1983, pp. 1-152.

123. STRAHM R. - "L'engagement de Roy A. Preiswerk dans la politique suisse de développement." Dans : PREISWERK R., A contre-courants. L'enjeu des relations interculturelles. Textes réunis et publiés par G. Rist. Lausanne, Ed. d'en bas (Coll. Nord-Sud), 1984, pp. 47-59.

124. WALSER R. - "Entwicklungshilfe und Ordnungspolitik. Die schweizerische Wirtschaft im entwicklungspolitischen Spannungsfeld." Schweizer Monatshefte, Nr. 10, 1983, S. 815-823.

125. WEISHAUPT P. - "Schweizer Intervention am Golf. Kriegsbeihilfe." Friedenszeitung, Nr. 35/36, Juli/August 1984, S. 32.

126. WICKER H. - "PSIND - Projekt zur Betreuung psychisch anfälliger Flüchtlinge. Ein Weg aus der Isolation ?" Mosquito, Nr. 3/4, Mai 1984, S. 29-30.

127. ZWAHLEN J. - “L'OCDE, la coopération internationale et la Suisse." Exposé présenté à l'occasion de l'ouverture des cours de l'Ecole des Hautes Etudes Commerciales de Lausanne, le 24.10.83. Paris, Délégation suisse auprès de I'OCDE,

\section{Autres articles}

128. “L'aide au développement est-elle une réalité ? / Ist die Entwicklungshilfe eine Realität ?" Forum avec la participation de F. Blankart, J.-F. de Dardel, E. Bernhardt, un représentant de "Mediswiss" et V. Arevalo. Zofingien (Genève), No 3 , mai 1983, pp. 228-253.

129. Beilage über die Wirtschaftsbeziehungen Schweiz - Indien. Schweizerische Hande/szeitung, Nr. 36, 1983.

130. "Les complicités au régime d'apartheid." Dans : Afrique du Sud : Du discours à la réalité. Genève, Centre Europe - Tiers Monde (Publicetim No 11), 1984, pp. 189 205.

131. "Referate, Broschüren und Leserbriefe für die Apartheid : Die Schweizer Freunde Südafrikas." Die WochenZeitung, Nr. 22, 3.6.1983.

132. "Les réfugiés africains à Genève. Attitude des médias, des Genevois, des Africains. Dossier." Echos africains, Journal trimestriel des associations culturelles africaines de Genève, No 1, juin-septembre 1984. 


\section{INSTITUTIONS}

\section{A. Confédération suisse}

\section{Messages et rapports du Conseil fédéral}

133. Message concernant l'accord international de 1982 sur le jute et les articles en jute, 17.8.1983. FF 1983 III 743. (All.)

Ce message figure à l'annexe 2 du Rapport sur la politique économique extérieure $1983 / 1$.

134. Message relatif aux nouvelles prorogations de la Convention sur le commerce du blé de 1971 de l'Accord international sur le blé de 1971, 7.9.1983. FF 1983 III 1173. (AII.)

135. Message concernant l'accord international de 1983 sur les bois tropicaux, 11.1.1984. FF 1984 I 573. (All.)

Ce message figure à l'annexe $14 \mathrm{du}$ Rapport sur la politique économique extérieure $1983 / 2$.

136. Message concernant la continuation de la coopération technique et de l'aide financière en faveur des pays en développement, 19.3.1984. FF 1984 II 1. (AII.)

137. Rapport sur la politique économique extérieure 1983/1, 17.8.1983. FF 1983 III 661. (All.)

138. Rapport concernant les mesures tarifaires prises pendant le 1er semestre 1983, 17.8.1983. FF 1983 III 775. (All.)

La première partie du rapport présente l'élargissement du schéma suisse des préférences tarifaires en faveur de cinq nouveaux pays classés dans la catégorie des pays les moins avancés.

139. Rapport concernant les mesures tarifaires prises pendant le 2e semestre 1983, 11.1.1984. FF 1984 I 89. (All.)

Le rapport traite de deux compléments au schéma suisse des préférences tarifaires en faveur des pays en développement.

140. Rapport sur les grandes lignes de la politique gouvernementale 1983-1987, 18.1.1984. FF 1984 I 153. (All.)

Voir notamment point 23 "La Suisse et les pays en développement", pp. 197-200.

141. Rapport sur la politique économique extérieure 1983/2, 11.1.1984. FF 1984 I 373. (All.)

142. Rapport sur les risques de l'endettement international, 12.3.1984. FF 1984 I 853. (All.)

143. Rapport concernant les problèmes globaux qui se posent sur le plan démographique, pour la sauvegarde des ressources et dans le domaine de l'environnement et leurs conséquences pour la Suisse. Office fédéral de la protection de I'environnement, Berne, 1984. (All.)

144. Effets économiques de l'aide publique au développement suisse. FF 1984 I 562. (All.)

Ce texte (résumé) figure à l'annexe II du Rapport sur la politique économique extérieure 1983/2. 
145. Evaluation du crédit mixte Egypte. FF 1984 I 559. (All.)

Ce texte (résumé) figure à l'annexe 10 du Rapport sur la politique économique extérieure 1983/2.

\section{Arrêtés fédéraux et ordonnances}

146. Arrêté fédéral concernant l'accord international de 1982 sur le jute et les articles en jute, 7.10.1983. FF 1983 III 1101. (All.)

147. Arrêté fédéral concernant l'accord international de 1983 sur les bois tropicaux, 23.3.1984. FF 1984 I 914. (All.)

148. Arrêté sur la participation de la Suisse aux Accords généraux d'emprunt du Fonds monétaire international, 4.4.1984. FF 1984 II 1185. (All.)

149. Arrêté fédéral concernant la conclusion de traités relatifs à la protection et à l'encouragement des investissements de capitaux. Prorogation du 24.6.1983. RO 1983 1432 (All.)

150. Arrêté fédéral concernant l'adhésion de la Suisse à la Banque africaine de développement, 19.12.1980. RO 1984 45. (All.)

151. Arrêté fédéral relatif à l'accord avec la République du Zaïre concernant l'indemnisation d'intérêts suisses, 17.6.1981. RO 1984 169. (All.)

152. Arrêté fédéral sur l'approbation de protocoles portant nouvelles prorogations de la Convention internationale sur le commerce de blé de 1971 de l'Accord international sur le blé de 1971, 13.3.1984. RO 1984 837. (All.)

153. Arrêté fédéral concernant I'adhésion de la Suisse aux Accords généraux d'emprunt du Fonds monétaire international, 14.12.1983. RO 1984 845. (All.)

154. Ordonnance concernant l'exécution de l'accord international sur le café de 1983, 19.9.1983. RO 1983 1278. (All.)

155. Ordonnance relative aux règles d'origine régissant l'octroi de préférences tarifaires aux pays en développement. Modification du 2.11.1983. RO 1983 1590. (All.)

156. Ordonnance fixant les droits de douane préférentiels en faveur des pays en développement. Modification du 5.12.1983. RO 1983 1768. (All.)

157. Ordonnance fixant les droits de douane préférentiels en faveur des pays en développement. Modification du 9.5.1984. RO 1984 665. (All.)

\section{Accords internationaux}

158. Accord portant création de la Banque africaine de dévelpppement en date du 4.8 . 1963 à Khartoum tel qu'amendé par la résolution 05-79 adoptée par le Conseil des gouverneurs le 17.5.1979. Conclu à Lusaka le 7.5.1982. Entré en vigueur pour la Suisse le 30.12.1982. RO 1984 46. (All.)

159. Accord international de 1983 sur le café. Conclu à Londres le 16.9.1982. Entré en vigueur provisoirement pour la Suisse le 1.10.1983. RO 1984 107. (All.)

160. Accords généraux d'emprunt du Fonds monétaire international. Conclus à Washington en 1962, amendés en 1983. Adhésion de la Suisse notifiée le 10.4.1984. RO 1984 847. (All.) 
161. Accord entre la Confédération suisse et la République de Panama concernant la promotion et la protection des investissements, 19.10.1983. FF 1984 I 567. (All.) Cet accord figure à l'annexe 13 du Rapport sur la politique économique extérieure $1983 / 2$.

162. Accord entre la Confédération suisse et la République du Zaïre concernant l'indemnisation des ressortissants suisses au Zaïre à la suite des mesures de zaïrianisation ou de radicalisation. Conclu le 8.10.1980. Entré en vigueur le 18.1.1984. RO 1984 170. (All.)

163. Echange de lettres du 17.11.1948 entre la Suisse et le Chili concernant la suppression réciproque du visa. Entré en vigueur le 1.1.1949. Cet accord a été en partie suspendu temporairement par décision du Conseil fédéral avec effet le 1.6.1984. RO 1984 519. (All.)

164. Protocole de 1983 portant septième prorogation de la Convention sur le commerce du blé de 1971. Entré en vigueur pour la Suisse le 24.5.1984. RO 1984838. (All.)

Publications et rapports de l'administration fédérale

165. Direction de la coopération au développement et de l'aide humanitaire : Rapport annuel 1983. Berne, DDA, 1984, 24. p. (All.)

166. La Direction de la coopération au développement et de l'aide humanitaire en 1983. Berne, DDA, septembre 1984, 134 p. (All.)

167. Mémorandum de la Suisse au Comité d'aide au développement. Rapport établi annuellement par la DDA et l'OFAEE à l'intention de l'Organisation de coopération et de développement économiques (OCDE).

168. Formes associatives (organisations d'épargnants, d'agriculteurs, d'artisans, de villageois, etc.). Contributions de C. Sidman-Steiner, J. Martin e.a. Entwicklung / Développement, No 15, septembre 1983, pp. 1-29. (All.)

169. Rôle de la technologie dans le développement. Contributions de P. Dudzik, S. Neumann e.a. Entwicklung / Développement, No 16, décembre 1983, pp. 1-38. (All.)

170. Populations et politiques démographiques. Contributions de C. Pfister, A. Mohr e.a. Entwicklung / Développement, No 17, juillet 1984, pp. 1-39. (All.)

170b. Rapport de la Commission de gestion au Conseil national sur le contrôle de l'efficacité de projets de développement au Népal du 23 août 1984, 27 p.

\section{B. Institutions privées}

Aktion Finanzplatz Schweiz - Dritte Welt, Bern

171. Die agressiven Aufsteiger. Materialien zur Schweizerischen Bankgesellschaft. Bern, März 1983, $33 \mathrm{~S}$.

172. Aktionsheft. Aktionsideen und Materialien zur Kampagne "Fluchtgeld nein Bankeninitiative ja !" Bern, 1983, 45 S. 
173. Argumentendossier. Fakten und Argumente zur Bankeninitiative aus entwicklungspolitischer Sicht. Bern, 1984, 79 S.

174. Finanzplatz-Informationen. Informationen zum Finanzplatz Schweiz und Argumente zur Bankeninitiative (erscheint 4-6 mal jährlich). Bern.

175. Fluchtgeld ist Fluchgeld. Ein Dossier zur Kapitalflucht in die Schweiz. Bern, 1983, $67 \mathrm{~S}$.

176. Verzell kei Bankemärli! Was den Schweizer Banken zur Bankeninitiative einfällt. Bern, 1984, $35 \mathrm{~S}$.

Association romande des Magasins du Monde, Lausanne

177. “1974-1984 : dix ans des magasins du monde", Bulletin No 13, janvier 1984.

Association suisse des banquiers / Schweizerische Bankiervereinigung, Basel

178. Les banques suisses et les problèmes des pays en développement. Bâle, 1982, 61 p. (All.)

Brot für Brüder, Basel

179. Mehr als ein Konto. Bankwesen und Bankengesetz : Reformen in Richtung Ausgleich und Entwicklung ? Basel, 1984, 55 S. (Fr.)

Caritas, Luzern

180. Not in den Slums - eine Herausforderung. Luzern, Caritas-Verlag, Oktober 1983, $34 \mathrm{~S}$.

Crédit Suisse / Schweizerische Kreditanstalt, Zürich

181. “Coopération économique avec le Tiers Monde." Série d'articles illustrant diverses formes de coopération au développement dues à I'initiative privée. Bulletin CS, No 8/9, septembre 1983, pp. 40-41, et No 1/2, février 1984, pp. 38-39. (All.)

Déclaration de Berne, Lausanne

182. “Les réfugiés : Droits de I'homme et Tiers Monde en Suisse." Vers un développement solidaire, No 68, septembre 1983, pp. 21-23.

183. "Contrats de BBC avec la Turquie et I'Afghanistan : entre chômage-businessmorale et risques financiers." Vers un développement solidaire, No 68, septembre 1983, pp. 24-26.

184. "Initiative sur les banques." Vers un développement solidaire, No 72 (numéro spécial), avril 1984, $27 \mathrm{p}$.

\section{Erklärung von Bern, Zürich}

185. “Beitritt der Schweiz zu den allgemeinen Kreditvereinbarungen : Armut und Gewalt." Dokumentation zum Rundbrief, Nr. 4, 1983, S. 9-20.

186. "Die Bankeninitiative : Solidarität vor Eigennutz." Dokumentation zum Rundbrief, Nr. 1, 1984, $12 \mathrm{~S}$. 
187. "Modell für Entwicklungsländer : Ferienparadies Schweiz ?" Dokumentation zum Rundbrief, Nr. 3, 1984, 12 S.

Fédération genevoise de coopération, Genève

188. Projets financés par l'intermédiaire de la Fédération genevoise de coopération entre 1977 et 1980 (1ère série de fiches, 1982) et entre 1980 et 1983 (2e série de fiches, 1984).

Informationsdienst Dritte Welt, Bern

189. Paternalismus statt Dialog. Schweiz, Medien und Dritte Welt. Bern, i3w, Dossier, Oktober 1983, $44 \mathrm{~S}$.

190. IWF und Dritte Welt. 40 Jahre Bretton Woods. Bern, i3w, Dossier, Juli 1984, $71 \mathrm{~S}$.

191. "Wachsendes Gewicht des BAWI in der schweizerischen Entwicklungspolitik." i3w-aktuell, Nr. 8, 1983, S. 9-11.

192. "Der Aerger mit den Mischkrediten." i3w-aktuell, Nr. 1, 1984, S. 2-4.

193. "Die internationale Seerechtskonvention." i3w-aktuell, Nr. 1, 1984, S. 7-10.

194. "Kompensationshandel." i3w-aktuell, Nr. 2, 1984, S. 6-10.

195. "Verschuldung der Entwicklungsländer : Was der bundesrätliche Bericht verschweigt." i3w-aktuell, Nr. 3, 1984, S. 4-6.

196. "Kontrolle beim Export gefährlicher Chemikalien notwendig." i3w-aktuell, Nr. 3 , 1984, S. 7-12.

197. “Sozialklauseln für Produkte aus ärmeren Entwicklungsländern. Das Beispiel Del Monte." i3w-aktuell, Nr. 4, 1984, S. 6-10.

198. "Bundesfinanzen und Entwicklungszusammenarbeit. Chronik einer politischen Auseinandersetzung." i3w-aktuell, Nr. 5, 1984, S. 2-5.

Information Tiers Monde, Service d' (i3m), Lausanne

199. Horlogerie suisse et Tiers Monde. Lausanne, i3m, Dossier No 14, mai 1984, 75 p.

200. Le Fonds Monétaire International et le Tiers Monde. Les 40 ans de Bretton Woods. Lausanne, i3m, Dossier No 15, juillet 1984, 70 p.

201. “La Suisse et les Accords Généraux d'Emprunts (AGE) : I'enjeu." Actualités i3m, No 6, 1983, pp. 5-11.

202. "La faim dans le monde : que fait la Suisse ?" Actualités i3m, No 6, 1983, pp. 12 15.

203. "Pétition pour l'aide au développement : un coup pour rien ?" Actualités i3m, No 7, 1983, pp. 5-7.

204. “Accords généraux d'emprunts ( $A G E$ ) : technique financière ou politique de développement ? Le point sur la question." Actualités i3m, No 8, 1983, pp. 6-9.

205. "Aide communale au développement : I'exemple de Vevey." Actualités i3m, No 1, 1984 , pp. 3-4.

206. "L'examen de I'aide suisse par I'OCDE." Actualités i3m, No 2, 1984, pp. 12-13. 
207. "Rapport du Conseil fédéral sur l'endettement international : une vision optimiste." Actualités i3m, No 3, 1984, pp. 4-5.

208. "Finances fédérales et coopération au développement : chronique d'une querelle politique." Actualités i3m, No 4, 1984, pp. 3-6.

209. "Juillet $84: 40$ e anniversaire des institutions de Bretton Woods. Et la Suisse ?" Actualités i3m, No 5, 1984, pp. 4-5.

Institut universitaire d'études du développement (IUED), Genève

210. Annuaire Suisse - Tiers Monde /Jahrbuch Schweiz - Dritte Welt 1983. Introduction de J. Forster. Genève, IUED, No 3, 1983, 361 p.

Justitia et Pax, Berne

211. La Suisse, place financière, et le Tiers-Monde. Résumé. Berne, Commission nationale suisse Justitia et Pax, Vol. 10, 1983, 45 p. (All., Ital.)

212. Développement et droits de l'homme. Normes éthiques pour la coopération au développement. Résumé. Berne, Commission nationale suisse Justitia et Pax, 1984, 52 p. (All., Ital.)

Movimento di Controinformazione zul sottosviluppo (MCS), Lugano

213. "La barca è piena... quasi per tutti." Nord-Sud, Informazione sul terzo mundo, No 23, set./ott. 1983, pp. 10-11.

214. "Le banche Svizzere ed il capitali in fuga da terzo mondo." Nord-Sud, No 26, mar./apr. 1984, pp. 1-14.

Parti Socialiste Ouvrier (POS) / Sozialistische Arbeiterpartei (SAP)

215. Eternit : Poison et domination. Une multinationale de I'amiante. Zurich, Ed. Veritas, 1983, 190 p. (All., Ital.)

Parti socialiste suisse (PSS) / Sozialdemokratische Partei der Schweiz (SPS), Bern

216. L'initiative sur les banques. Dossier. Berne, 1984, 64 p. (All.)

Schweizerische Kontaktstelle für Angepasste Technik (SKAT), St. Gallen

217. Angepasste Technik und die Dritte Welt. Ein Arbeitsbericht der SKAT. Hrsg. von J.-M. Baumer, U. Heierli, U. Meier und U. Rentsch. St. Gallen, Institut für Lateinamerikaforschung und Entwicklungszusammenarbeit an der Hochschule St. Gallen, 1983,146 S.

Shell (Suisse), Zurich

218. La Suisse et le pétrole. Parution annuelle. (All.)

Solidaritätskomitee für Afrika, Asien und Lateinamerika / Arbeitsgruppe 3. Welt (SKAAL/AG3W), Bern

219. Dossier "Schweiz-Trikont." Enthält kurze Informationen über Finanzbeziehungen, Handel, Entwicklungshilfe, Multis, Diplomatie usw. und einen entwicklungspolitischen Kommentar. In : Mosquito. 
220. Mosquito (erscheint zehnmal jährlich). Siehe Punkt 1. B.

Swissaid, Bern

221. Schweizerische Hilfe für Entwicklungsländer / Aide suisse aux pays en développement. (Annuaire statistique.)

World Wildlife Fund Schweiz (WWF)

Schweizerischer Bund für Naturschutz (SNB)

Schweizerische Gesel/schaft für Umweltschutz (SGU)

222. Biozid-Report Schweiz. Schadstoffe in unserer Umwelt : Situation und Lösungsansätze. Aarau, Sauerländer Verlag, 1984, $641 \mathrm{~S}$.

Autres travaux collectifs

223. Waffenplatz Schweiz. Beiträge zur schweizerischen Rüstungsindustrie und Waffenausfuhr. Hrsg. vom Tagungssekretariat "Für das Leben produzieren." Bern, Arbeitsgemeinschaft für Rüstungskontrolle und ein Waffenausfuhrverbot, Basel, Forum für praxisbezogene Friedensforschung, Zürich, Schweizerischer Friedensrat, 1983, $185 \mathrm{~S}$. 